\title{
LEI DE INOVAÇÃO E PESQUISA ACADÊMICA
}

\section{INNOVATION LAW AND ACADEMIC RESEARCH}

\author{
Elisabeth Adriana Dudziak ${ }^{1}$; Guilherme Ary Plonski ${ }^{2}$ \\ ${ }^{1}$ Escola Politécnica da Universidade de São Paulo - EPUSP - São Paulo - Brasil \\ elisabeth.dudziak@poli.usp.br \\ ${ }^{2}$ Escola Politécnica da Universidade de São Paulo - EPUSP - São Paulo - Brasil \\ plonski2@usp.br
}

\begin{abstract}
Resumo
O objetivo do trabalho é analisar o modelo brasileiro de inovação, buscando estabelecer a ligação entre a teoria, a prática e as intervenções no processo empreendidas pelo poder público, a partir das políticas adotadas. A abordagem teórica (perspectiva analítica) do tema desenha-se sobre os paradigmas da ciência, tecnologia e inovação: linear, sistêmico e complexo. O foco normativo recai sobre o marco legal da Lei de Inovação n. 10.973 e os possíveis impactos de sua adoção no meio acadêmico. Interessa-nos principalmente examinar o eixo de flexibilização das atividades dos pesquisadores, mobilidade e relações de trabalho nas universidades públicas. Do ponto de vista da práxis acadêmica, elegeu-se como objeto de estudo o Departamento de Engenharia de Energia e Automação Elétricas (PEA) da Escola Politécnica da Universidade de São Paulo (USP). Na fronteira teórica mundial relativa à $C, T \& I$ há indícios de instauração do paradigma complexo, no qual a sustentabilidade, a inovação sustentável e a inteligência distribuída têm papel preponderante. As políticas públicas brasileiras relativas à $C, T \& I$ evidenciam alinhamento ao paradigma sistêmico competitivo, com foco em P\&D nas empresas. Do ponto de vista institucional, pode-se afirmar que está em curso na USP um processo de transição conduzido principalmente no meso-nível dos processos administrativos. Com referência à práxis acadêmica de pesquisa observada no PEA, há indícios de transição ao paradigma sistêmico complexo. Conclui-se que no momento não há possibilidade de evolução harmoniosa dos sistemas de C,T\&I devido à falta de alinhamento entre teoria, prática e políticas.
\end{abstract}

Palavras-chave: teoria da inovação; lei de inovação; comunidades de pesquisa; pesquisa.

\section{Introdução}

Em sentido geral, o objetivo deste trabalho é analisar o sistema brasileiro de C,T\&I (Ciência, Tecnologia e Inovação), buscando estabelecer a ligação entre a teoria, a prática e as políticas implementadas através do exame do possível impacto da Lei de Inovação Federal n. 10.973 sobre a comunidade acadêmica de pesquisa e suas atividades. Entende-se que somente o alinhamento entre fundamentos teóricos (paradigmas), direcionamento político e a relação de ambos com as atividades de pesquisa pode promover uma evolução harmoniosa dos sistemas de ciência, tecnologia e inovação. Parte-se de um referencial teórico que considera distintos paradigmas científicos, 
tecnológicos e inovativos: paradigma linear, paradigma sistêmico e abordagem complexa. Examina-se o cenário brasileiro relativo às políticas de C,T\&I, com foco na Lei de Inovação 10.973. Por fim, estuda-se o impacto das políticas e especificamente da aplicação da Lei de Inovação sobre as atividades e o ambiente de pesquisa em um grupo de pesquisadores de uma universidade pública. O estudo de caso pretende aprofundar os conhecimentos acerca desse impacto, considerando distintas visões e paradigmas subjacentes às práticas de pesquisa desses pesquisadores.

Como premissa fundamental, considera-se que os processos de ciência, tecnologia, inovação e desenvolvimento das nações se estruturam a partir de determinadas visões de mundo, paradigmas que podem ser reconhecidos na teoria, na prática e nas políticas implementadas (KUHN, 1970). Nesse sentido, observa-se que há uma inerente ligação entre a teoria da C,T\&I, a práxis acadêmica de pesquisa e as intervenções no processo empreendidas pelo poder público (SMITHS; KUHLMAN, 2004). Apenas o alinhamento entre teoria, prática e política produz evolução harmoniosa desses sistemas.Assim sendo, da relação dialética e evolutiva entre a teoria de C,T\&I, a política e as práticas acadêmicas de pesquisa, pretende-se aprofundar os conhecimentos acerca da coerência ou não entre o modelo pretendido (teoria/abstração subjacente à política) e o modelo apropriado pela comunidade científica (teoria/abstração subjacente às práticas de pesquisa acadêmica).

Elegeu-se como objeto de estudo de caso o Departamento de Engenharia de Energia e Automação Elétricas da Escola Politécnica da Universidade de São Paulo. O estudo, baseado em entrevistas e documentos departamentais, busca estabelecer a visão de mundo dos pesquisadores, concepções, como gerenciam suas agendas de pesquisa, a quais condicionantes institucionais estão submetidos, como percebem as políticas e a lei de inovação.

\section{Perspectiva Analítica}

Nas últimas décadas, avanços importantes têm sido observados quanto à teoria da inovação, ciência e tecnologia: a natureza, processos e avaliação das atividades de C,T\&I, as políticas e estratégias de intervenção evoluíram. Se o desenvolvimento da teoria se dá essencialmente no esteio da indicação de relação entre variáveis ou conceitos, novas teorias surgem todos os dias em resposta a indagações não respondidas pelas teorias anteriores, preenchendo e desenvolvendo o quadro de conhecimentos existente. Observando a trajetória de mudanças nos estudos sobre os sistemas de C,T\&I, três paradigmas se distinguem: paradigma linear, sistêmico e complexo.

O paradigma linear da ciência, tecnologia e inovação desenvolveu-se no pós-guerra como síntese de progresso das nações (BUSH, 1945). O direcionamento axiomático, compartimentado, seqüencial e redutor do processo de inovação não se sustentou por muito tempo. A regulação estrita 
tampouco solucionou o distanciamento entre a comunidade científica e a sociedade. O fim do paradigma linear teve como maior conseqüência o declínio da abordagem neoclássica e o despertar para a abordagem evolutiva dos sistemas. Os mecanismos de diversificação e seleção, a dependência das trajetórias de aprendizado, definiram ao longo dos anos o entendimento da inovação como processo de busca e aprendizado interativo (CASSIOLATO; LASTRES, 1998).

A ascensão do paradigma sistêmico e sua consolidação permitiram compreender que as instituições e organizações evoluem a partir de mecanismos de seleção e variedade, segundo uma racionalidade técnica. São únicas, porém imersas no sistema. Como conseqüência, sua performance é dependente do desempenho do sistema como um todo, particularmente em função da qualidade (eficiência) dos sub-sistemas (mercado, $\mathrm{P} \& \mathrm{D}$, usuários, intermediários e rede institucional que os suporta). A ênfase cognitiva está explícita nos modelos prescritivos e na ênfase da regulação orientada pelo mercado.

Sucessivas gerações de modelos sistêmicos foram sugeridos pelos estudiosos, com especial destaque aos Sistemas Nacionais de Inovação (DOSI, 1982; FREEMAN, 1982; LUNDVALL, 1992; NELSON, 1993); o modo 2 de produção de conhecimento (GIBBONS et al, 1994); o Quadrante de Pasteur e a pesquisa duplamente orientada (STOKES, 1997); Porter (1990) e o diamante nacional; a Hélice Tripla e a interação entre governo, universidade e empresa (ETZKOWITZ; LEYDERSDORFF, 2002).

A abordagem complexa apresenta-se agora como movimento de superação paradigmática da abordagem sistêmica, logrando ir um passo além. Relacionada à sustentabilidade co-evolucionária de sistemas locais, regionais, nacionais e supra nacionais, a abordagem complexa está baseada na constante re-estruturação dialética entre ambiente e sistema que, no limite, são iguais. A emergência do pensamento da complexidade foi antes de tudo uma tentativa de entender a realidade, o ser no mundo, uma vez que a ciência tradicional não é mais suficiente para explicar os fenômenos (MORIN e Le MOIGNE, 2000).

A questão da sustentabilidade é recorrente devido à inerente implicação na co-evolução e eco-regulamentação, a partir do contínuo desenvolvimento e aprendizado interativos. Nesse sentido, a interação em redes conduz à necessidade de reformular "o contrato social da ciência, da tecnologia e da inovação". Inteligência distribuída, energia distribuída, inovação distribuída, P\&D distribuídos, economia distribuída indicam a existência de arquiteturas horizontais dos sistemas.

Atividades complexas são realizadas simultaneamente por um número elevado de elementos conectados (artefatos tecnológicos e/ou seres humanos), fronteiras institucionais e/ou geográficas cotidianamente são superadas. Importam o aprendizado interativo e o comportamento dinâmico das empresas. Um novo paradigma industrial estaria se estruturando a partir do conceito de solução sustentável industrial (industrialised sustainable solution). Deste modo, tem-se a evolução de um 
capitalismo predatório para um capitalismo natural. O capital natural e uma política de energia economicamente eficiente somam-se ao redesenho dos processos de produção, de retorno e fechamento dos fluxos de materiais e serviços para promover maiores ganhos de duração longa.

Alguns autores já admitem a constituição de sociedades inovativas. Instauram-se mecanismos de governança multi-nível (multi-level governance), concebida com base em complexos jurídicos flexíveis que integram dimensões verticais e horizontais. $\mathrm{Na}$ linha da abordagem de multi-níveis de análise e articulação (detail complexity), desenvolve-se o trabalho de Fuller, Warren e Argyle (2005), e Geels e Kemp (2001). Desenvolvem pesquisas metodológicas cujas bases encontram-se na ciência da complexidade e no construcionismo social. Caracterizam a inovação como a emergência de irregularidades logo legitimadas a partir de movimentos e imperativos sociais, tecnológicos e de mercado (regimes). Rycroft e Kash (1999) e Metcalf (2003) desenvolveram estudos semelhantes em linhas intermediárias entre os modelos sistêmico e complexo, re-dimensionando os aspectos econômico e tecnológico.

Vivencia-se a ecologia da ação amparada em uma racionalidade substantiva, que supõe a compreensão da relação estreita entre pensamento (teoria) e ação, entre individual e coletivo, entre política e vida cotidiana (regulação reflexiva). O Quadro 1 a seguir, confronta os diferentes aspectos e características de cada paradigma, buscando sintetizar suas principais características.

Quadro 1 - Confronto entre os paradigmas linear, sistêmico e complexo

\begin{tabular}{|c|c|c|c|}
\hline $\begin{array}{c}\text { Paradigma } \\
\text { Característica }\end{array}$ & Linear & $\begin{array}{c}\text { Sistêmico } \\
\text { Hélice Tripla }\end{array}$ & Complexo \\
\hline Relação & Linear & $\begin{array}{c}\text { Conectiva e } \\
\text { Interseccional }\end{array}$ & $\begin{array}{l}\text { Todo e partes / Inteligência } \\
\text { distribuída }\end{array}$ \\
\hline Papel do Estado & Provedor & Controlador / Regulador & Inovador/Emancipador \\
\hline Organização & $\begin{array}{l}\text { Tradicional/ } \\
\text { Burocrática }\end{array}$ & $\begin{array}{l}\text { Knowledge Organization } \\
\text { / Learning Organization }\end{array}$ & Complex Organization \\
\hline $\begin{array}{c}\text { Conceito de } \\
\text { desenvolvimento }\end{array}$ & $\begin{array}{l}\text { Desenvolvimento } \\
\text { endógeno }\end{array}$ & $\begin{array}{c}\text { Competitividade/ } \\
\text { Desenv. Econômico } \\
\text { Competit/Micro e Meso } \\
\text { Desenv. } \\
\end{array}$ & Desenvolvimento sustentável \\
\hline Recursos & Infraestrutura & $\begin{array}{c}\text { Recursos Humanos / } \\
\text { Capital humano e capital } \\
\text { social }\end{array}$ & $\begin{array}{l}\text { Sociedade e meio ambiente } \\
\text { capital natural }\end{array}$ \\
\hline Ator central & Universidade & $\begin{array}{c}\text { Empresa / Universidade- } \\
\text { Governo-Empresa }\end{array}$ & Multi-atores / Sociedade \\
\hline Regulação & Estrita & Flexível & Reflexiva \\
\hline
\end{tabular}

\section{Políticas de C,T\&I e a Lei de Inovação 10.973}

Constata-se que as políticas de inovação também se transformaram ao longo do tempo, com base na co-evolução de distintos sistemas e regimes. Produzida da amálgama entre política científica, tecnológica e industrial, a política de inovação evoluiu em função do aprendizado da aplicação da teoria na prática e da avaliação dos resultados percebidos. Nesse sentido, a política de 
C,T\&I atual volta-se à abordagem complexa na medida que é um processo no qual multi-atores interagem, em multi-níveis e multi-fases de aprendizado.

A nova geração de política de inovação assume que somente a governança dos sistemas de $C, T \& I$ será capaz de promover o desenvolvimento sistêmico e sustentável, a partir de ações integradas e coordenadas. Um Estado reflexivo atua como mediador nas relações entre distintos e cada vez mais diversificados grupos de interesse, baseando suas ações em uma inteligência distribuída.

No caso do Brasil, a centralidade da ação da mudança ainda está no Estado. Deste modo, observa-se uma inclinação à reatividade, tanto por parte das empresas, organizações, universidades e demais instituições de pesquisa, quanto por parte da sociedade como um todo. A comunidade acadêmica tem exercido pressão no sentido de prospectar caminhos de atuação e intervenção do poder público em direção ao paradigma sistêmico. Porém, do ponto de vista societal, não foram ainda percebidas potenciais mudanças.

$\mathrm{O}$ conceito de inovação subjacente à política nacional brasileira enfatiza o binômio $\mathrm{P} \& \mathrm{D}$ baseado em macro-estratégias e sistemas de financiamento (foco no ex-ante), e visa o aumento da competitividade e valor agregado das exportações. Deste modo, observa-se que a centralidade das políticas governamentais está no fortalecimento do setor produtivo empresarial de média e alta tecnologia.

O sistema de C,T\&I brasileiro é fortemente influenciado por fatores externos como por exemplo a incerteza da macroeconomia, fragilidade institucional, infra-estrutura débil ou incerta (serviços básicos de energia que falham, sistemas de comunicação ultrapassados), falta de conscientização sobre a importância da inovação (ausência de cultura de inovação), aversão ao risco por parte das firmas, ausência de liderança e empreendedorismo, carência de instrumentos de políticas públicas capazes de apoiar os processos de inovação. Como conseqüência, alguns padrões tornam-se persistentes: assimetrias organizacionais, concentração da inovação em poucas empresas ou setores, pequenas mudanças incrementais são as mais freqüentes formas de inovação, a tecnologia (maquinário) é geralmente importada.

Entretanto, começam a ser percebidos sinais de uma apropriação complexa da questão a partir das ações de prospecção, mediação e construção de agendas intencionais (surgidas a partir do diálogo entre os distintos atores, pela agregação heterogênea entre opiniões individuais e experiências no repertório, e na agenda coletiva). A ênfase na articulação política entre os atores e entre os distintos níveis de intervenção tem direcionado aos instrumentos de governança dos sistemas de inovação. A visão sistêmica e contextual aparece quando se contemplam áreas como o desenvolvimento social, tecnologias sociais, energias renováveis, gestão ambiental. O conceito de inovação amplia-se: de evento tecnológico a fenômeno sociotécnico emergente que resulta de um 
processo de aprendizado, e que pode ou não gerar benefícios eqüitativos e/ou ecologicamente corretos. Mas é preciso direcioná-la à sustentabilidade.

Fazendo uma retrospectiva, é possível observar que os esforços empreendidos pelo poder público em relação à $\mathrm{C}, \mathrm{T} \& \mathrm{I}$ têm seguido caminho semelhante ao experimentado em outros países. No que se refere à integração, o desafio real é buscar uma coesão política em torno do tema, bem como aumentar a coordenação de atividades ministeriais e consolidar o conceito de trabalho em rede, incluindo outros atores além da universidade-governo-empresa, como é o caso das associações, organizações não governamentais, incubadoras, cooperativas, arranjos produtivos locais, entre outros.

Nesse contexto surge a Lei de Inovação Federal n. 10.973, ensejando promover a ligação entre universidade, empresa e governo. Aprovada em 2004 e somente regulamentada em 2005, a Lei de Inovação ainda precisa ser disseminada. Através da Lei, o governo brasileiro sinaliza para:

- O despertar da sociedade para a inovação;

- A constituição de um aparato institucional de estímulo à inovação;

- Legitimação do caráter estratégico da inovação na agenda de desenvolvimento nacional;

- Intenção de valorização das atividades de pesquisa e articulação entre pesquisadores e empresas;

- Sinalização da necessidade de aprimorar competências para a realização de grandes empreendimentos;

- Direcionamento à cooperação entre universidade e empresa.

Entretanto, não há até o momento nenhuma mudança substancial no quadro geral nacional em função da regulamentação da lei. Não houve um aumento perceptível de articulação entre atores, tampouco as empresas têm assumido riscos e implementado comportamento inovador. A Lei de Inovação, ao focar as atividades de P\&D nas empresas, sinaliza para uma condução subjacente no sentido de considerar as atividades científicas como adjuntas às atividades de tecnologia e inovação e, portanto, às empresas. Tal postura reflete um viés utilitarista de tratamento da questão tanto da educação quanto da ciência brasileira. O foco excessivo nos atributos organizacionais relativos à competitividade e produtividade revelam um direcionamento à concepção de inovação como fator de acumulação econômica e não como estratégia de desenvolvimento da nação. Mas como será que tudo isso se reflete no ambiente acadêmico?

\section{Atividades de Pesquisa e o Impacto da Lei de Inovação}

O ambiente de trabalho acadêmico vem sofrendo um inexorável processo de enfraquecimento. Neste sentido, tornam-se relevantes os impactos gerados pela ciência na sociedade e o compromisso do cientista com a sociedade. 
Hoje a universidade pública está sujeita a uma série de forças que têm direcionado sua atuação. Encontra-se pressionada a adotar uma nova identidade e experimenta um processo de desinstitucionalização, em função de heteronomias e subordinação ao mercado, o que a direciona à internacionalização, massificação de ensino e delineando a emergência da universidade empreendedora. Entretanto, do ponto de vista da comunidade de pesquisa e seu trabalho, prevalece a cultura científica e seus princípios. A organização de colegiados e a governança, em geral baseada no modelo de Déficit Científico, contribuem para a consolidação de valores tradicionais como a autonomia, liberdade de pesquisa, neutralidade da ciência e racionalidade.

Se é no tecido de relações sociais que se constroem as prioridades de pesquisa, também aí se incorporam os interesses econômicos e políticos, sinais de relevância no sentido desejado são emitidos, e terminam por delimitar os campos de relevância, as áreas-problema que devem ser os objetos de trabalho dos pesquisadores. Tais campos são submetidos a avaliações de qualidade, cujos critérios e valores são definidos pelo próprio tecido. (DAGNINO; THOMAS, 2001). É nesse contexto que se fortalecem as interações entre ciência e indústria, universidade e empresa (PLONSKI, 1995).

A colaboração entre universidade e empresa em termos de pesquisa e desenvolvimento tecnológico tem história antiga, remontando ao século XIX (GUSMÃO, 2002). A natureza e o alcance dos mecanismos oficiais de interação variam muito de país para país. Segundo Velho, Velho e Saenz (2004), as empresas estão investindo mais em P\&D, tanto em seus próprios laboratórios quanto nas universidades. Quando se associam às universidades, buscam novas idéias, talentos e novos procedimentos essenciais às suas operações, prospectando possibilidades de inovação que deverão ocorrer dentro de quatro ou cinco anos. De todo modo, as universidades não substituem os departamentos de P\&D das empresas, mesmo porque atuam de forma diversa (a empresa, mais pragmática e técnica, a universidade, mais exploratória).

Sob a perspectiva sistêmica, a política industrial e um conjunto de leis de C,T\&I também têm contribuído para a formalização desta relação. Novas estruturas têm sido criadas, modalidades de interação se diversificam, criando condições para a sustentabilidade dos sistemas, ambientes e comunidades de pesquisa. A mobilidade profissional e de conhecimentos é a cada dia mais valorizada em função do potencial de difusão de aprendizado e inovação que carrega. Nesse sentido, deve ser estimulada também pela universidade.

Se, de fato, ultrapassamos a fase formativa da institucionalidade brasileira de C,T\&I, como bem pode ser demonstrado pela evolução histórica, trata-se agora de consolidar a estrutura em torno de consensos, buscando a transição para uma nova institucionalidade mais reflexiva, integrada e emancipada. 


\section{Universidade de São Paulo como lócus da práxis acadêmica de pesquisa}

Estudos recentes demonstram a centralidade da pesquisa brasileira na universidade pública, consubstanciada em ilhas de excelência alicerçadas em seu capital humano e social, e baseadas em redes. Há um acompanhamento das tendências internacionais em termos de flexibilização das relações de trabalho e volatividade crescente de vínculos. As estruturas de poder nestas instituições são polissêmicas, apresentando forte diferenciação horizontal, alta autonomia e baixa interdependência. Com relação à interação com outros atores e a mobilidade, persiste o perfil isolado de pesquisador acadêmico, exceção experimentada na Engenharia (BALBACHEVSKY, 2005).

A natureza das atividades de pesquisa realizadas nas universidades brasileiras, apesar de expandida, permanece ainda condicionada predominantemente ao ambiente acadêmico, seguindo agora quatro eixos de carreira: docência, pesquisa, gestão (atividades administrativas) e extensão (consultorias e cursos). Em termos institucionais, os ideais originais de universidade ainda fazem parte do imaginário sócio-cultural da sociedade brasileira, principalmente no que se refere à universidade pública. Observa-se que o regime científico brasileiro apresenta-se desarticulado dos demais regimes sociotécnicos, evidenciando a necessidade de se promover reformas institucionais de cunho regulativo (estatutos, regimentos, procedimentos), cognitivo (modelos e paradigmas) e normativo (papéis e valores).

A Lei da Inovação surge no cenário como instrumento de flexibilização de trocas e autorização de exercício simultâneo de atividades afetando principalmente as universidades públicas federais.

Nesse contexto, a Universidade de São Paulo (USP) vem se organizando institucionalmente para o desenvolvimento das atividades de pesquisa e inovação. A partir da análise de suas raízes históricas, observa-se que sua concepção original baseia-se na contradição fundamental entre o modelo francês e o modelo alemão, suplantados parcialmente na década de 60 pelo modelo norteamericano (PAULA, 2002). Muitos dos ideais acadêmicos humboldtianos e inclinações hierárquicas francesas ainda estão presentes, principalmente em sua missão, nos discursos, rituais, enfim, na cultura subjacente à prática dos docentes-pesquisadores.

Analisando os processos de decisão institucional na Universidade, constata-se um direcionamento à modalidade de colegiado, ou comunidade de homens cultos (DAGNINO; GOMES, 2002). Observa-se uma tendência geral a um processo de decisão que ocorre por consenso, com o exercício de autoridade legitimado com base na competência, experiência e lealdade à instituição, muito mais que na hierarquia, embora esta última seja estritamente aplicada. 
As relações que se estabelecem junto à sociedade são guiadas pela manutenção do prestígio da instituição e de seus profissionais.

A evolução da instituição foi marcada por uma intensa relação contextual e social, tanto brasileira quanto paulista. Hoje, em geral apresenta expressiva interação com o segmento empresarial, articula-se facilmente com os demais atores universitários, agências e órgãos governamentais, tanto estaduais quanto federais. Para tanto, conta com uma estrutura poderosa que inclui as Pró-Reitorias (em especial as Pró-Reitorias de Pesquisa e de Cultura e Extensão), e outras estruturas interfaciais como as Fundações e o CIETEC (Centro Incubador de Empresas Tecnológicas) da USP.

Suas bases estatutárias evoluíram e se transformaram ao longo do tempo, buscando a sintonia possível com a realidade e as demandas sócio-econômicas, a partir do gerenciamento de convênios e contratos, possibilidade de exercício simultâneo de atividades e afastamento de pesquisadores. Apesar da estruturação, predominam as interações informais e/ou pontuais com a sociedade e as empresas em geral: contratos de consultoria e pesquisa sob encomenda acontecem envolvendo indivíduos, mais que grupos ou laboratórios de pesquisa. A formalização da interação ocorre quando se desenvolvem projetos com grandes empresas como a Petrobrás e a CVRD.

A Universidade conta também com estruturas formais de facilitação de transferência de conhecimento, a Agência USP de Inovação e do NUDI-EP (Núcleo de Inovação da Escola Politécnica), organismos que possuem assessoria especializada para a prospecção de oportunidades, divulgação de chamadas públicas, desenvolvimento de projetos e gestão de propriedade intelectual. A estas estruturas somam-se as Fundações, interlocutores indispensáveis, uma vez que agilizam as trocas entre a Universidade e o setor privado. Importantes também têm sido as avaliações individuais e institucionais, uma vez que o governo estadual e a sociedade de forma geral têm exercido pressão, sobretudo no que se refere à qualidade de interação com o público e a necessidade de transparência de alocação dos recursos públicos na Universidade.

No que se refere às comunidades de pesquisa, predomina o modo 1 de atuação acadêmica: o conhecimento está mais restrito à comunidade, é disciplinar e a produção científica está concentrada na instituição (GIBBONS, 1994). O ambiente de trabalho é hierárquico e estável, baseado em consensos e comunicado internamente por vias predominantemente informais e difusas.

Em algumas áreas porém, principalmente na Engenharia, o comportamento é distinto, mais flexível e direcionado à resolução dos problemas sociais. $\mathrm{O}$ direcionamento ao modo 2 de produção de conhecimento (idem ibidem) pode ser sentido no aumento o número de projetos conjuntos entre universidades-empresas-agências de fomento, e atividades de cooperação entre indústria e universidade, empresa e universidade, incubadoras e parques tecnológicos, determinando como evidência o aumento da diversidade de fontes de financiamento das pesquisas e redes de 
cooperação. A Pró-Reitoria de Pesquisa, a Agência USP de Inovação e o NUDI-EP têm trabalhado intensamente na prospecção e inclusão da Universidade nesses projetos.

Estruturalmente, entretanto, existe uma histórica resistência à mudança, ancorada no exercício da autoridade acadêmica e científica. Apesar dos esforços empreendidos pelos órgãos centrais da Universidade, muito ainda pode ser feito. A articulação entre as universidades paulistas estaduais poderia ser maior. Não há sinais de integração entre bancos de dados informacionais de pesquisas, currículos ou complementaridade de cursos. Ações sistêmicas estratégicas são desejáveis.

As políticas de pesquisa não são explícitas, tampouco há um plano diretor. Diretrizes são importantes mas não há metas. A falta de transparência nos processos e na prestação de contas das unidades é outro aspecto que contribui para a manutenção do corporativismo e dos entraves existentes. Os mecanismos de interface e divulgação do portfólio de pesquisas não estão explícitos, não se detectam sinais de acolhimento e direcionamento à aproximação com a sociedade, mesmo porque a questão da comunicação institucional e social é ainda bastante controversa e deficiente. A flexibilização de atividades tanto dos pesquisadores quanto da própria Universidade são questões por discutir. A internacionalização é um movimento forte que tende a se difundir. A preocupação com a inclusão de alunos e o aumento no número de vagas são outras frentes prioritárias. A valorização do pesquisador acadêmico é outro ponto a ser tratado.

A Lei de Inovação não está na pauta das atividades institucionais. Ainda que haja esforços por parte da Agência USP de Inovação, esta não parece ser uma área prioritária para a Universidade. Neste contexto, examinaremos o caso do grupo de pesquisadores da Engenharia.

\section{O Caso PEA - Departamento de Engenharia de Energia e Automação Elétricas da Escola Politécnica da USP}

Energia, desenvolvimento e meio ambiente são elementos fortemente inter-relacionados. Para os países em desenvolvimento, o tema da energia é de fundamental importância. Nesse sentido, o despertar e crescente conscientização dos impactos ambientais dos empreendimentos não só na área de energia, como nas áreas industrial, extrativa, agrícola, etc, conduzem à necessidade de redefinição do significado de desenvolvimento.

Aproximadamente 57\% da energia utilizada no Brasil é de energia limpa e renovável, sendo $39 \%$ de hidroeletricidade e 18\% de biomassa. A base energética brasileira, portanto, é renovável e portadora de menor potencial de agressão ao meio ambiente. Está em andamento o Plano Decenal de Expansão de Energia Elétrica 2006-2015. Em fase de consolidação está o Plano Nacional de 
Energia 2030, criado com o intuito de orientar tendências e estabelecer alternativas de expansão do sistema nas próximas décadas.

Outro programa importante é o Programa Nacional de Universalização do Acesso e Uso da Energia Elétrica - "Luz para Todos", cujo objetivo é levar energia elétrica para a população do meio rural. Além destas ações, o Ministério de Minas e Energia tem investido em projetos em conjunto com os demais países da América Latina, sem deixar de lado as energias renováveis.

A gestão dos processos e da política de inovação na área é essencial. Sobretudo, existe a necessidade de implementar ações de planejamento distribuído, integrando energia, ambiente e políticas de desenvolvimento, bem como os distintos atores e suas práticas: governo, agências do setor, agências de fomento e financiamento, universidades, indústrias, organizações e comunidades.

\subsection{As pesquisas em energia na USP}

$\mathrm{Na}$ área de energia, a USP desenvolve atividades de pesquisa em numerosas vertentes. A começar pelo Programa Interunidades de Energia , que tem como linhas de pesquisa:

a) Planejamento Integrado de Recursos;

b) Análise Econômica Institucional de Sistemas Energéticos;

c) Fontes Renováveis e não Convencionais de Energia;

d) Energia, Meio Ambiente e Sociedade;

e) Redes Elétricas, Equipamentos e Qualidade de Energia.

Essas iniciativas são financiadas majoritariamente por órgãos de fomento e empresas públicas nacionais. Outra importante iniciativa é o Programa PURÊ, relacionado à economia de energia. A Universidade também tem transformado parte do esgoto produzido pela residência e pelo restaurante universitários em energia elétrica. Pesquisadores da Escola Politécnica, do Instituto de Química, do Instituto Oceanográfico, do Instituto de Astronomia, Geofísica e Ciências Atmosféricas, do Instituto de Geociências, do Instituto de Física, do Instituto de Química de São Carlos e do Instituto de Eletrotécnica e Energia, entre outras unidades, estão aliando forças para melhor explorar seu potencial de trabalho na área.

\subsection{O Departamento de Engenharia de Energia e Automação Elétricas}

O PEA - Departamento de Engenharia de Energia e Automação Elétricas - faz parte da Grande Área Elétrica da Escola Politécnica da USP, juntamente com outras três grandes áreas Civil, Mecânica e Química. É responsável pela formação de estudantes de engenharia com ênfase 
em energia e automação.

As atividades de ensino encontram-se voltadas à formação de profissionais capazes de atuar tanto tecnicamente quanto politicamente. A forte ligação com o ambiente empresarial é característica marcante no corpo discente de pós-graduação, constituindo-se em fonte recorrente de interação com esse meio.

\subsubsection{A organização do trabalho de pesquisa}

O departamento está estruturado em três coordenadorias departamentais, cada qual experimentando certo grau de autonomia, no que se refere às decisões que envolvem suas áreas de atuação: a coordenadoria de pós-graduação e pesquisa/extensão (envolvendo os grupos de pesquisa), a coordenadoria de ensino de graduação (envolvendo as áreas didáticas) e a coordenadoria administrativa (contemplando as estruturas de apoio), conforme definido no PGPEA de 1999.

No Plano de Gestão do PEA (PGPEA) ficou estabelecida a política de gestão departamental orientada para o apoio aos grupos de pesquisa. Também foram determinadas as metas de desempenho para estes grupos e para o departamento como um todo, a partir do oferecimento das adequadas condições: infra-estrutura física, recursos tecnológicos e recursos humanos de apoio às atividades de pesquisa. Os grupos de pesquisa são responsáveis pelas disciplinas de pós-graduação, pelos projetos de pesquisa e atividades de extensão.

Como grupo de pesquisadores, o departamento está estruturado como uma burocracia profissional. Os times são formados por profissionais altamente qualificados, que possuem um grau de autonomia bem elevado, formando de fato um grupo de especialistas. O controle é alcançado a partir de consensos e regras comuns (profissionalismo). Evidenciam-se ainda assim traços de burocracia profissional, principalmente em nível departamental, privilegiando-se a disciplina típica do profissional de ensino e pesquisa. A comunidade é aderente aos valores acadêmicos. $\mathrm{O}$ apoio administrativo é estruturado a partir de uma burocracia hierárquica tradicional, sem representatividade substancial no processo decisório da instituição.

Esta estrutura favorece as interações com outros atores justamente a partir das especialidades do grupo. Pontos fortes são as habilidades técnicas e o padrão profissional de trabalho. Nesse sentido, a estrutura de governança técnico-científica incorpora modos de cooperativos de negociação, reunindo atores empresariais, governamentais e universitários. No que se refere à interação com outros atores, o modelo de governança de déficit científico é o mais utilizado.

\subsubsection{Concepções, modelos mentais e percepção das políticas de C,T\&I}

O conceito internalizado de ciência no grupo exibe contornos positivistas, identificados com 
o racionalismo. O modo clássico de pensar do cientista: ciência como verdade universal, positiva e passível de controle. A ciência praticada é produto do grau de diferenciação social. O conceito de inovação ainda está bastante atrelado à invenção e, nesse sentido, depreende-se que prevalece um entendimento linear do processo.

Ainda assim, ciência e tecnologia parecem se desenvolver no departamento na justa medida de sua interação com a sociedade. Em termos gerais, os pesquisadores realizam a chamada pesquisa duplamente orientada: ao entendimento e ao uso, uma vez que além dos projetos conjuntos com as empresas e outras instituições, os pesquisadores também publicam artigos e trabalhos científicos, almejando ainda o reconhecimento internacional (STOKES, 1997). Há consenso entre os docentes que a relevância e a qualidade das pesquisas estão diretamente relacionadas aos resultados e efeitos gerados nas empresas e na sociedade. Segundo os próprios pesquisadores, a avaliação baseada nas publicações científicas é um aspecto importante, mas não especialmente o mais adequado quando se trata da Engenharia. Nesse sentido, a diversificação de critérios de avaliação é um caminho promissor e as atividades cooperativas são incentivadas.

O trabalho de pesquisa deve se ajustar a uma agenda repleta de outras atividades, onde se destaca o ensino. A gestão dá suporte às ações departamentais e reforça a autonomia nas decisões de alocação de pessoas e recursos. A relação entre o departamento e as empresas acontece tanto informal quanto formalmente, dependendo da duração e amplitude das atividades de cooperação planejadas. A viabilização de tal interação só é possível porque há um alinhamento entre

julgamentos e expectativas de pesquisadores e empresários, pela convergência de interesses. Definitivamente, o trabalho do grupo está ancorado em uma reputação construída ao longo dos anos e é suportado pela legislação federal de repasse de recursos às pesquisas pelas empresas.

Barreiras institucionais principais ao pleno desenvolvimento das atividades de pesquisa são a burocracia e a falta de transparência dos processos. Estruturas de interação como a Agência USP de Inovação e o NUDI-EP são elogiadas como iniciativas necessárias e positivas, mas não são percebidas ações efetivas ou importantes destes órgãos em relação ao departamento.

As políticas governamentais relativas à área de energia e automação elétricas são percebidas como importantes, porém carentes de pragmatismo e coordenação. A Lei de Inovação não exerce qualquer impacto nas atividades do grupo, sendo quase desconhecida pelo grupo. $\mathrm{O}$ grau de conhecimento do conteúdo da lei é baixo. Nesse sentido, a intenção de apropriação da lei fica comprometida.

\section{Conclusões}

Este trabalho teve como objetivo amplo aprofundar os conhecimentos acerca do modelo brasileiro de C,T\&I (Ciência, Tecnologia e Inovação), buscando estabelecer a ligação entre a teoria, 
a prática e as intervenções no processo empreendidas pelo poder público, a partir das políticas implementadas e o impacto dessas políticas no ambiente acadêmico de pesquisa.

A revisão de literatura evidencia uma tendência à abordagem complexa em todos os campos do conhecimento. Desenha-se pois a iminência de uma transformação de paradigmas, considerando questões de amplitude mundial como o desenvolvimento sustentável e a sustentabilidade ambiental, social, econômica, cultural, entre outras. Percebe-se que os processos complexos envolvem perspectivas de longo prazo, consistentes com ações integradas que envolvem multi-atores, multiníveis e multi-fases de mudança. A transição do paradigma atual para um paradigma de sustentabilidade pode ser alcançada se houver agentes indutores de mudança.

Se na fronteira teórica mundial relativa à $\mathrm{C}, \mathrm{T} \& \mathrm{I}$ há indícios de instauração do paradigma complexo, em muitos países latino-americanos traços de direcionamento teórico ao paradigma sistêmico são recentes. Têm como centralidade a dimensão tecnológica e a empresa como lócus preferencial da inovação. Entretanto, ainda não se pode falar em sistema de C,T\&I latinoamericano, uma vez que predomina na região uma situação de desarticulação entre os distintos atores, evidenciando o alinhamento das práticas ao paradigma linear.

No caso do Brasil, a forte influência exercida pela comunidade científica, aliada às pressões externas da globalização e do neoliberalismo, determina no país um direcionamento da C,T\&I ao paradigma sistêmico evolucionista, seguindo as tendências observadas nos países centrais, e isso tem se refletido nas políticas públicas.

Diante desse cenário, observa-se a necessidade de, antes de tudo, retornar ao básico e tratar as incongruências entre o modelo institucional e jurídico existente, e a situação desejada. A revisão da função do Estado passa necessariamente pela diminuição dos entraves burocráticos, e a excessiva ênfase no ex-ante e no controle de processos, realizando um caminhar em direção à aferição de resultados e impactos das intervenções governamentais gerados na sociedade. Além disso, para ser efetivo, o compromisso com a inovação deve ser assumido como primeira diretriz governamental, ação transversal e prioridade nacional. Nesse contexto, apenas a aprovação da Lei de Inovação não é suficiente, uma vez que, sem uma real mudança de paradigmas acadêmicos, não haverá apropriação de novos instrumentos de fomento à inovação.

Dentro de uma perspectiva sistêmica complexa, as trajetórias e relações entre os regimes tecnológico, sócio-cultural, científico, político e ambiental também necessitam estar alinhados, para que de fato o regime de inovação se estabilize e possa ser difundido, atingindo o macro-nível. Estes regimes também se encontram desalinhados, expondo conflitos de interesse e falta de uma inteligência regimental.

Argumenta-se que não existe de fato um explícito direcionamento político à inovação e ao desenvolvimento sustentável, enquanto projetos nacionais acadêmicos. A política industrial 
recentemente aprovada mostra-se incapaz de estabelecer uma pragmática direção e resultados a serem alcançados. O mercado apóia-se muito no Estado e não constrói um regime autônomo. Textualmente as leis, e em particular a Lei de Inovação, apresentam incongruências e falta de pragmatismo, com definição obtusa.

Também o regime científico brasileiro apresenta-se desordenado, necessitando promover reformas institucionais de cunho regulativo (estatutos, regimentos, procedimentos), cognitivo (modelos e paradigmas) e normativo (papéis e valores). Do ponto de vista das instituições de ciência e tecnologia e, em particular, das universidades, constata-se concentração das atividades de pesquisa nas instituições públicas.

A comunidade acadêmica tem exercido pressão no sentido de prospectar caminhos de atuação e intervenção do poder público em direção ao paradigma sistêmico. Porém, do ponto de vista da própria universidade, não foram ainda percebidas potenciais mudanças. O conservadorismo universitário ainda é barreira a ser vencida. $\mathrm{O}$ encorajamento às parcerias entre universidade $\mathrm{e}$ empresa, inter-empresas, projetos conjuntos, parques tecnológicos, parece ter encontrado terreno promissor na engenharia. Neste caso, assinala-se como referência teórica o modelo da hélice tripla. Apesar de sua orientação à articulação, o Estado ainda atua como principal ator e financiador.

Diante desse quadro, definir o regime de C,T\&I pretendido pela Universidade de São Paulo torna-se tarefa primária. Os aspectos regulativos (conjunto de leis, estruturas, procedimentos), normativos (deveres, papéis, valores) e cognitivos (modelos de realidade, crenças, paradigmas) devem estar alinhados no próprio regime. Esse alinhamento até o momento não existe.

Em termos institucionais, os ideais originais de universidade ainda fazem parte do imaginário sócio-cultural da sociedade brasileira, principalmente no que se refere à universidade pública.

Com referência à práxis acadêmica de pesquisa observada no Departamento de Engenharia de Energia e Automação Elétricas (PEA), constata-se a apropriação de instrumentos de gestão eficiente. Como ponto de partida, esta consideração por si mesma já direciona a visão da atividade de pesquisa como ação sistêmica. Por outro lado, sendo a comunidade do PEA a um só tempo produto e produtora da Universidade de São Paulo, sua diferenciação tem limites bem claros. O engajamento institucional emoldura suas práticas e relações.

Nesse sentido, muitos dos princípios positivistas científicos ainda estão presentes e subjacentes aos conceitos de ciência, pesquisa e inovação. Por outro lado, a pesquisa duplamente orientada é praticada rotineiramente. O modo 1 de produção de conhecimento ainda está presente mas o modo 2 (GIBBONS et al, 1994) é efetivamente utilizado uma vez que há desenvolvimento de pesquisas conjuntas com empresas e comunidades, embora esta seja ainda uma atividade adjunta. 
A ligação entre pesquisa, desenvolvimento sustentável, e a relação entre energia e sustentabilidade social evidenciam um direcionamento ao pensamento complexo. Por todos estes fatores, há indícios de transição ao paradigma sistêmico complexo nas atividades do grupo.

Entendendo que apenas o alinhamento entre teoria de C,T\&I, política de C\&T e de Inovação e a práxis acadêmica de pesquisa leva a uma evolução harmoniosa dos sistemas, conclui-se que, devido à teoria estar direcionada ao paradigma complexo, as políticas orientadas ao paradigma sistêmico competitivo e as práticas observadas no PEA estarem em transição para o paradigma complexo, no momento não há possibilidade de alinhamento e evolução harmoniosa dos sistemas de C,T\&I. Não há, portanto, real impacto da política de inovação atual (Lei de Inovação) na práxis acadêmica.

\begin{abstract}
The purpose of this work is to analyze the Brazilian innovation model, to establish the link between theory, practices and the interventions in the process undertaken by the public power, as from the policies adopted. The theoretical approach (analytical perspective) of the subject is drawn on the paradigms of science, technology and innovation: linear, systemic and complex. The normative focus is on the Law of Innovation n. 10,973 and possible impacts of its adoption into the academic environment. The focus is on research activities flexibility, mobility and work relations in public universities. The Department of Energy and Electric Automation Engineering (PEA) of the Engineering School of the University of Sao Paulo (USP) was chosen as a study object. In the world-wide theoretical border of studies in $S, T \& I$, there are indications of establishing the complex paradigm, in which sustainability, sustainable innovation and distributed intelligence have a preponderant role. The Brazilian public policies in S,T\&I have evidences on the alignment to the competitive systemic paradigm, focused on the companies' $R \& D$. From the institutional point of view, it can be said that a transition process is in course at USP, led mainly in the mid-level of the administrative processes. Regarding the academic research praxis observed in PEA, there are indications of transition to the complex systemic paradigm. It is concluded that, at the moment, harmonious evolution of the Science, Technology and Innovation systems is not possible, due to lack of alignment among theory, practices and policies.
\end{abstract}

Keywords: innovation theory; innovation law; research communities; research

\title{
Referências
}

BALBACHEVSKY, E. Profissão acadêmica no Brasil: contexto institucional e condições de trabalho (1992-2003). São Paulo, 2005. Tese (Livre-Docência). Faculdade de Filosofia, Letras e Ciências Humanas da Universidade de São Paulo, 2005.

CASSIOLATO, J.E.; LASTRES, H.M.M. Inovação, globalização e novas políticas de desenvolvimento industrial e tecnológico. Nota Técnica 21/98. Rio de Janeiro, IE/UFRJ, 1998.

DAGNINO, R.; THOMAS, H. Planejamento e políticas públicas de inovação: em direção a um marco de referência latino-americano. Planejamento e Políticas Públicas, n. 23, 2001. 
DOSI, G. Technological paradigms and technology trajectories. Research Policy, v. 11, p. 147-162, 1982.

cross ${ }^{\text {ref }}$

ETZKOWITZ,H.; LEYDESDORFF, L. The Triple Helix-University-Industry-Government Relations: A Laboratory for

Knowledge Based Economic Development. EASST Review, v. 14, n.1, p. 14-19, 1995. http://users.fmg.uva.nl/lleydesdorff/th1/index.htm Acesso em: 23 Jan. 2006.

FREEMAN, C. The economics of industrial innovation. London: Pinter Publishers, 1982.

FULLER, T.; WARREN, L.; ARGYLE, P. Towards a complex explanation of innovation as order creation through emergence. In: COMPLEXITY, SCIENCE \& SOCIETY CONFERENCE, Liverpool, 2005. Papers. Liverpool: The University of Liverpool, $2005 . \quad$ Disponível em: http://www.liv.ac.uk/ccr/2005_conf/subject_areas/mngt_files/papers/toward_complex_explanation_etc.pdf Acesso em: 26 Jun. 2006.

GEELS, F.W.; KEMP, R. Transitions, transformations and reproduction: dynamics in socio-technical systems. In: DYNAMICS OF INDUSTRY AND INNOVATION:ORGANIZATIONS, NETWORKS AND SYSTEMS, Copenhagen, Denmark, 2005. Proceedings. Copenhagen, 2005.

GIBBONS, M. et al. The new production of knowledge: the dynamics of science and research in contemporary societies. London: Sage, 1994.

GUSMÃO, R. Práticas e políticas internacionais de colaboração ciência-indústria. Revista Brasileira de Inovação, v. 1, n.2, Julho/Dezembro 2002. p. 327-360.

LUNDVALL, B. National Systems of Innovation: towards a theory of innovation and interactive learning. London: Pinter Publishers, 1992.

METCALF, J.S. Equilibrium and evolutionary foundations of competition and technology policy: new perspectives on the division of labor and the innovation process. Revista Brasileira de Inovação, v.2, n. 1, Jan/Jun 2003. [Idéias originais de 1995].

MORIN, E.; LE MOIGNE, J.L. A inteligência da complexidade.2.ed. São Paulo : Peirópolis, 2000.

NELSON, R. R. (ed.). National Systems of Innovation: a comparative study. Oxford: Oxford University Press, 1993.

PAULA, M. F. C. USP e UFRJ. A influência das concepções alemã e francesa em suas fundações. Tempo Social; Rev. Sociol. USP, v.14, n.2, p. 147-161, outubro de 2002.

PEA. Departamento de Engenharia de Energia e Automação Elétricas da Escola Politécnica da Universidade de São Paulo. Auto-avaliação e síntese do Plano de Metas do Departamento. 2003. São Paulo: 2003.

. PGEA - Plano de Gestão do PEA. São Paulo, 1999.

PLONSKI, G.A. Cooperação universidade-empresa: antigos dilemas, novos desafios. Revista da USP, n.25, 1995. p. 32-41.

PORTER, M.E. The competitive advantage of nations. New York: McMillan, 1990.

RYCROFT, R.; KASH, D.E. Innovation policy for complex technologies. Issues in Science and Technology, v. 16, n. 1, Fall 1999, p. 73-74.

SMITS, R.; KUHLMAN, S. The rise of systemic instruments in innovation policy. International Journal of Foresight and Innovation Policy, v.1, n.1/2, p. 4-32, 2004.

cross ${ }^{\text {ref }}$

STOKES, D. O quadrante de Pasteur: ciência básica e inovação tecnológica. Campinas: UNICAMP, 2005. Do original de 1997.

VELHO, L.; VELHO, P.; SAENZ, T.W. P \& D nos setores público e privado no Brasil: complementares ou substitutos? Parcerias Estratégicas, n. 19, p. 87-127, 2004. 
Dados dos autores:

\section{Elisabeth Adriana Dudziak}

Escola Politécnica da Universidade de São Paulo

Divisão de Biblioteca

Chefe de Seção Técnica

Av. Prof. Luciano Gualberto Trav. 3, n. 158

05508-970 - São Paulo - SP

(11) 3091-9024

e-mail: elisabeth.dudziak@poli.usp.br

\section{Guilherme Ary Plonski}

Escola Politécnica da Universidade de São Paulo

Departamento de Engenharia de Produção

Professor Titular

Av. Prof. Almeida Prado, Trav. 2, n. 128

05508-070 - São Paulo - SP

(11) 3091-5363

e-mail:plonski2@usp.br

Recebido para publicação em: 01/02/2008

Aceito para publicação em: 29/02/2008 